\title{
Mit System zum Büro der Zukunft
}

\author{
Im Hinblick auf eine nachhaltige Entwicklung rücken Systeminnovationen \\ in den Blickpunkt. Sie gehen über Verbesserungen bestehender Produkte und \\ Dienstleistungen hinaus und verbinden technologische Veränderungen mit \\ organisatorischen und sozialen Neverungen.
}

D

Von Wilfried Konrad, Dirk Scheer und Ursula Tischner ie Innovationsforschung unterscheidet Innovationstypen nach Reichweite. Diese grundlegende Unterscheidung grenzt inkrementelle von radikalen Innovationen ab. Inkrementelle Innovationen sind relativ geringe Veränderungen von Prozessen und Produkten und finden in allen Wirtschaftszweigen mehr oder weniger kontinuierlich statt. Sie sind oft Ergebnis von Learning by Doing auf Basis von Erfahrung oder Learning by Using aufgrund von Nutzervorschlägen.

Radikale Innovationen treten hingegen diskontinuierlich auf und sind üblicherweise das Ergebnis gezielter FuE-Aktivitäten im technologischen Bereich. Sie sind ungleich über Wirtschaftszweige und Zeit verteilt. Als Beispiele werden Nylon, Atomkraft und die Pille oder die Entwicklung des Transrapid als Abkehr vom Rad-Schiene-System und der Umstieg vom Explosionsmotor zur Brennstoffzelle genannt (1). Gemeinsam ist diesen Innovationen, dass sie kombinierte Produkt-, Prozess- und organisatorische Veränderungen beinhalten.

\section{Systeminnovationen - Hoffnung für Nachhaltigkeit?}

Inkrementelle wie radikale Innovationen besitzen ökologisches Entlastungspotenzial - und stoßen dabei dennoch schnell an Grenzen. Häufig werden beispielsweise die mit schrittweisen Verbesserungen verbundenen Entlastungspotenziale durch Wachstumseffekte überkompensiert. Auch technologisch orientierte radikale Innovationen greifen hier zu kurz. Denn die Konstatierung eines direkten Zusammenhangs zwischen technisch-ökonomischer und ökologischer respektive Nachhaltigkeitsreichweite übersieht, dass über diese nicht zuletzt die Nutzungsmöglichkeiten und Anwendungskontexte der Innovation entscheiden.

Zugleich ist zu vermuten, dass auch nutzungsorientierte Veränderungen alleine keine Entlastungspotenziale bereithalten, die dem Nachhaltigkeits-
Vor diesem Hintergrund wurden im Projekt verschiedene Konzepte für bürobezogene Systeminnovationen entwickelt, die derzeit detailliert ausgearbeitet werden und die nach Projektende Anfang 2005 von den beteiligten Unternehmen in den Markt eingeführt werden sollen. Aus dem Kreis der erarbeiteten Systeminnovationskonzepte werden im Folgenden zwei näher dargestellt.

\section{Autarkes Fassaden- Baukastenelement}

postulat genïgen. Die Untersuchung von entsprechenden Fällen, wie die Nutzung von Waschsalons oder Mietskiern, ist hier instruktiv (2).

Im Hinblick auf die Erzielung von relevanten Nachhaltigkeitseffekten geht es also um weitreichende Neuerungen in beiden Dimensionen, also um komplexe Verknüpfungen von wissenschaftlich-technischen, organisatorisch-institutionellen und sozialen Neuerungen, die in der innovationsbezogenen Nachhaltigkeitsdebatte als Systeminnovationen bezeichnet werden (3).

\section{- Das nachhaltige Büro}

Die Entwicklung von Systeminnovationen im Bürobereich steht im Zentrum des Projekts „Das nachhaltige Büro" (4). Die Arbeitswelt der Zukunft wird vermehrt durch Bürotätigkeiten und weniger durch produzierende Tätigkeiten gekennzeichnet sein. Bereits heute aber ist klar, dass mit Büroausstattung und -betrieb erhebliche Umweltbelastungen verbunden sind, die insbesondere den Bereichen Gebäude, Verkehrsaufkommen, elektro- und informationstechnische Infrastruktur, Beleuchtung, Lüftung und Klima, Bürogeräte und Büromöblierung zuzurechnen sind. Zum anderen gibt es zahlreiche Hinweise auf weitreichende Veränderungen der herkömmlichen Büroarbeit, die auf eine Verlagerung von der ortsbestimmten, eher materiellen, reproduzierenden Einzelarbeit zur eher virtuellen, eher ortsunabhängigen, eher kreativen Teamarbeit deuten (5).

\section{Wussten Sie schon,}

dass der ökom Verlag nicht nur Zeitschriften sondern auch Bücher verlegt? Wir helfen Ihnen gerne weiter, wenn Sie Ihre Forschungsergebnisse, Dissertation oder Tagungsdokumentation veröffentlichen möchten. Unser aktuelles Buchprogramm finden Sie unter www.oekom.de/verlag/german/books/index.htm Hier können Sie auch unser kostenloses Verlagsprogramm 2004 bestellen. 
Der systemische Charakter der Innovation betrifft sowohl Planung wie Umsetzung. Voraussetzung für die erfolgreiche Entwicklung und Umsetzung der Systeminnovation ist die interdisziplinäre Zusammenarbeit von Materialtechnikern, Planern, Zulieferern und Herstellern etc. und die Herausbildung neuer Arbeits- und Produktionsprozesse. So wird die Fassadensanierung bei Altbauten mit dem autarken Fassaden-Baukastenelement nicht mehr vorrangig auf dem $\mathrm{Zu}$ sammenspiel verschiedener Handwerker wie Fensterbauern, Wasser- und Heizungsinstallateuren, Malerbetrieben etc., basieren, sondern von auf die neue Technologie spezialisierten Installateuren ausgeführt werden, die alles aus einer Hand liefern.

Mögliche ökologische und wirtschaftliche Nachhaltigkeitsvorteile der Systeminnovation liegen etwa im Erhalt und der Aufwertung von Bausubstanz und dem dadurch reduzierten Flächenverbrauch, in reduzierten Energieverbräuchen durch effiziente Fassadenmontage und bessere Dämmung, in der Nutzung umweltfreundlicher Materialien, in den im Vergleich zu einer herkömmlichen Sanierung niedrigeren oder allenfalls gleichen Investitionskosten oder in den durch die modulare Bauweise niedrigen Entsorgungskosten.

\section{- Dezentrale Büro-Box}

Hier geht es um die Bedürfnisse von modernen Büroarbeitenden, die viel reisen und immer weniger Zeit im eigentlichen Büro verbringen. Die Systeminnovation Dezentrale Büro-Box ist eine Infrastruktur, die Bedürfnisse des modernen Büroarbeiters ökonomisch und ökologisch effizient sowie sozialverträglich und befriedigend erfüllt. Es handelt sich um eine unabhängige, mobile Kapsel, die sich an den unterschiedlichsten Orten aufstellen lässt und eine komplette Büroinfrastruktur mit modernster Technik zur Verfügung stellt.

Die Boxen haben alles, was die Nutzer für ihre Büroarbeit und Besprechungen benötigen und lassen sich individuell an die Bedürfnisse der jeweiligen Nutzer anpassen. Das System wird von lokalen Servicepartnern wie Reinigungskräften und Getränkelieferanten versorgt und bietet somit eine Kombination von Büroraum und dazugehörigen Dienstleistungen. Vorzugsweise wird die Büro-Box an Verkehrsknotenpunkten und stark frequentierten Orten aufgestellt.

Die Systeminnovation zielt auf einen neuen Markt für moderne, nutzergerecht gestaltete und lokali- sierte Büroräume, die hochmobilen Wissensarbeitern eine attraktive Alternative zur Nutzung feststehender Bürogebäude bieten soll. Die ökologische Nachhaltigkeit der Büro-Box wird durch die Einsparung an neuen, flächenintensiven Immobilien, die effiziente Gestaltung von Stromversorgung, Heizung, Kühlung und Licht, die Nutzung von Solartechnik und Regenwasser, sowie eine langlebige Konstruktion angestrebt. Sie soll sich den sozialen Bedürfnissen der Nutzer hinsichtlich Ergonomie, Nutzung von Informations- und Kommunikationstechnologien, Licht, Raumatmosphäre etc. anpassen und kann zur Kostenreduktion durch Office-Sharing beitragen.

\section{- Resümee}

Die beiden diskutierten Beispiele zeigen, dass Systeminnovationen komplexe sozio-technische Konstellationen mit weitreichendem Veränderungspotenzial sind. Zentral für ihre Entstehung ist die Kooperation verschiedener, bislang weitgehend unverbundener Akteure - hier von Unternehmen und Architektur- und Ingenieurbüros über Verbände bis hin zu Verbraucherschutzorganisationen und Gewerkschaften. Die Einbindung unterschiedlicher Perspektiven auf den Bürobereich ergibt sich dabei zum einen aus der für Systeminnovationen notwendigen Integration verschiedener Einflussgrößen, zum anderen im Hinblick auf das Ziel der Generierung nachhaltiger Systeminnovationen. Beides ist aufgrund begrenzter Kompetenzen, Ressourcen, Kontakte etc. von Einzelakteuren oder einer homogenen Akteursgruppe wie zum Beispiel Büromöbelhersteller nicht zu leisten.

Mit Blick auf das Thema Nachhaltigkeit zeigt sich, dass Systeminnovationen keineswegs per se nachhaltig sind, wie dies in Teilen der Diskussion zumindest suggeriert wird. Zum anderen gilt, dass es sich bei den identifizierten Nachhaltigkeitsaspekten um hypothetische Potenziale handelt, die sich in der konkreten Anwendung der Systeminnovationen realisieren lassen können - oder eben nicht.

Vor diesem Hintergrund hat im aktuellen Prozess der detaillierten Ausgestaltung der vorliegenden bürobezogenen Systeminnovationskonzepte die Diskussion der mit ihnen verbundenen Nachhaltigkeitsdimensionen eine besondere Bedeutung. Derzeit werden die oben betrachteten Systeminnovationen mit Hilfe orientierender Ökobilanzen analysiert. Dabei ist zu beachten, dass Nachhaltigkeit nicht wie beispielsweise technische oder ökonomische Leistungsmerkmale in die Systeminno- vationen eingebaut werden kann. Vielmehr gilt in Anlehnung an Grunwald, dass ihre „Gestaltung unter Nachhaltigkeitsaspekten (...) einen ständigen Lernprozess (bildet), orientiert durch das normative Leitbild der Nachhaltigkeit, in dem über Gestaltungsziele, Realisierungsoptionen und die zukünftige Einbettung (...) in die Gesellschaft diskutiert wird" (6).

\section{Anmerkungen}

(1) Freeman, C./ Perez, C.: Structural crisis of adjustment, business cycles and investment behaviour. In: Dosi, G./ Freeman, C./ Nelson, R./ Silverberg, G./ Soete, L. (Eds.): Technical Change and Economic Theory. London/ New York 1988, pp. 38-66 sowie Klemmer, P./ Lehr, U./ Löbbe, K.: Umweltinnovationen - Anreize und Hemmnisse. Berlin 1999.

(2) Hirschl, B./ Konrad, W./ Scholl, G./ Zundel, S.: Nachhaltige Produktnutzung. Sozial-ökonomische Bedingungen und ökologische Vorteile alternativer Konsumformen. Berlin 2001.

(3) Konrad, W./ Nill, J.: Innovationen für Nachhaltigkeit. Schriftenreihe des IÖW 157/01, Berlin 2001 sowie Rocha, C./ Brezet, H./ Peneda, C.: The development of productoriented environmental management systems (POEMS). The Dutch experience and a case study. Delft 1999.

(4) Das Projekt wird vom Bundesministerium für Bildung und Forschung gefördert und gemeinsam von IÖW und econcept in Kooperation mit einer Vielzahl an Branchenakteuren durchgeführt. Info: www.nachhaltigesbuero.de

(5) Hube, G.: Arbeits- und Büroformen heute und morgen. Fraunhofer-Institut für Arbeitswirtschaft und Organisation, Stuttgart 2003.

(6) Grunwald, A.: Ein ambivalentes Verhältnis. Nachhaltigkeit und Schlüsseltechnologien. In: Ökologisches Wirtschaften, Nr. 6/ 2003, S. 13-14.

Die Autorlnnen

Dr. Wilfried Konrad und Dirk Scheer sind wissenschaftliche Mitarbeiter im Forschungsfeld Ökologische Produktpolitik am Institut für ökologische Wirtschaftsforschung (IÖW). Ursula Tischner ist Leiterin der Agentur für Nachhaltiges Design econcept und Assistenzprofessorin für Sustainable Design an der Design Academy Eindhoven.

Kontakt: IÖW, Büro Heidelberg, Bergstr. 7, 69120 Heidelberg. Tel. 06221-649160, Fax 06221-27060, E-Mail: mailbox@heidelberg.ioew.de; econcept, Mainzer Str. 23, 50678 Köln. Tel. 0221-4202676, Fax 0221-4202674, E-Mail: v.tischner@econcept.org 
(c) 20I0 Authors; licensee IÖW and oekom verlag. This is an article distributed under the terms of the Creative Commons Attribution Non-Commercial No Derivates License (http://creativecommons.org/licenses/by-nc-nd/3.o/), which permits unrestricted use, distribution, and reproduction in any medium, provided the original work is properly cited. 